\title{
Viewpoint
}

\section{Bad press for peer review: is it deserved?}

\section{Emma Palmer-Cooper}

Department of Psychiatry, University of Oxford; emma.palmer-cooper@psych.ox.ac.uk; DOI: 10.20316/ESE.2016.42.017

Peer review has recently been getting some bad press. There is ongoing debate across the sciences about its validity and usefulness, with many believing it is slow, costly, biased, and inconsistent. ${ }^{1}$ In the last few years alone several high-profile cases in the media have highlighted these downfalls. One such example is the reaction on Twitter to a PLOS reviewer's suggestion that male authors be added to a manuscript examining gender bias in academia, which was written by two female researchers (Figure 1). Another is the revelation that a number of researchers had systematically submitted bogus articles and faked peer review, ${ }^{2,3}$ and the retraction of over 120 articles published by Springer and IEEE over "gobbledygook" articles that had nonetheless passed peer review. More lighthearted criticisms of the process have included multiple online collections of the best and worst comments left by reviewers.

Fiona Ingleby
बFionalngleby

Reviewer's conclusion: we should get a man's name on MS to improve it (male colleagues had already read it) (2/4)

It would probably also be beneficial to find one or two male biologists to work with (or at least obtain internal peer review from, but better yet as active co-authors), in order to serve as a possible check against interpretations that may sometimes be drifting too far away from empirical evidence into ideologically biased assumptions.

Figure 1. Tweet by Fiona Ingleby, highlighting biased comments from anonymous reviewer.

However, I and many other scientists feel that despite its downfalls, peer review is a useful process that benefits publications and researchers alike - when executed properly.

As a reviewer, I have enjoyed the process of reading and critically analysing new manuscripts from my field of research. I have taken the comments I make and applied them to my own writing to ensure it is prepared to the same standards. I have likewise found most peer review comments I have received helpful and feel they have led to the publication of better quality articles.

One of the main problems with the review process stems from peers themselves, either not taking the process seriously, or not understanding what is required of them. There are various articles available that set out how to write a good peer review. A simple search returns a plethora of hits that all point to a similar method and structure for the review. There are also dedicated pages available on publisher's websites. So why don't all reviewers stick to this? Simply, because they are not required to. Journal editors often do not ensure reviewers adhere to these guidelines, or merely give feedback that comments are unhelpful, vague or inappropriate.

Some journals have tried to tackle these issues by asking reviewers to additionally rate different aspects of a manuscript on Likert scales, which provides some limited feedback and indicates where improvements could be made. However, the most important aspect of reviewing, at least for articles requiring revisions, is the comments. Nonetheless, the degree to which comments can improve a manuscript will vary. Though a reviewer writing "The writing and data presentation are so bad that I had to leave work and go home early and then spend time to wonder what life is about" 4 is amusing, in reality it is not helpful to the author.

The problem faced when trying to enforce guidelines is that peer review is commonly unpaid and anonymous. I enjoy the process of peer review, and thus try to be as constructive as I can with my feedback, but for some academics this alone is not enough. With no incentive for reviewers to be constructive, laziness, sarcasm, and poor attitude can follow.

Some suggest that peer review should be abolished altogether, I however, disagree. Instead, reforms are required to improve upon an imperfect but necessary system.

A starting point for reform would be to remove reviewer blinding, forcing peers to either be constructive in their comments or decline the request. Elsevier took this idea one step further, announcing they would trial publishing reviews of accepted articles in article format on ScienceDirect, naming the reviewers. They also now recognise quality reviewers with a Certificate of Excellence. While some believe this change would result in reviewer bias, such as junior academics holding back criticisms of a more senior author, it again falls to the reviewers to "play by the rules", or decline to act as a reviewer. Further, serious concerns or queries one may have about a manuscript, but not wish to publish, can still be sent confidentially to the Editor. Other ways in which journals could improve the peer review process would be to cap how long they wait for responses and comments from reviewers, as long delays can hinder the publication process. Some have even suggested that journals might pay reviewers to ensure quality. However, in an age where open access is a major aim while simultaneously keeping other costs down, this option is looking unlikely.

Peer review is not perfect, and therefore deserves some of the criticism it has received to date. However, without evaluation from others in the field, many published articles would be unclear, unfounded or incorrect. Reform is required to ensure confidence in peer review, but it is up to us as academics and reviewers who take on the role to do so responsibly, and the journals and their editors to keep those who do not in check.

\section{References}

1 Smith, R. Peer review: a flawed process at the heart of science and journals. Journal of the Royal Society of Medicine. 2006;9(4):178-182.

2 Haug, C. J. Peer-review fraud-hacking the scientific publication process. New England Journal of Medicine. 2015;373(25):2393-2395.

3 Bohannon, J. Who's afraid of peer review. Science. 2013;342(6154).

4 Referees' quotes - 2010 Environmental Microbiology. 2010;12:3303-

3304. doi:10.1111/j.1462-2920.2010.02394.be 